www.mdpi.com/journal/molecules

Article

\title{
Modulation of Huntingtin Toxicity by BAG1 is Dependent on an Intact BAG Domain
}

\author{
Jan Liman ${ }^{1,2, \dagger}$, Kamila Sroka ${ }^{3, \dagger}$, Christoph P. Dohm ${ }^{1,2}$, Sebastian Deeg ${ }^{1,2}$, Mathias Bähr ${ }^{1,2}$ and \\ Pawel Kermer ${ }^{1,2, *}$
}

1 Deptment of Neurology, University of Göttingen, Robert-Koch Str. 4037075 Göttingen, Germany

2 DFG-Research Center for Molecular Physiology of the Brain (CMPB), Humboldtallee 23, 37075

Göttingen, Germany; E-Mails: jliman@gwdg.de (J.L.); cdohm@gwdg.de (C.P.D.);

mbaehr@gwdg.de (M.B.)

3 Merz Pharmaceuticals, R\&D CNS, In vitro Pharmacology, Eckenheimer Landstrasse 100, 60318

Frankfurt, Germany, E-Mail: kamilasroka@yahoo.co.uk (K.S.)

$\dagger$ These authors contributed equally to this work.

* Author to whom correspondence should be addressed; E-Mail: pkermer@gwdg.de;

Tel.: +49-551-396356; Fax: +1-111-111-112.

Received: 11 August 2010; in revised form: 16 September 2010 / Accepted: 19 September 2010 /

Published: 28 September 2010

\begin{abstract}
Huntington's disease, one of the so-called poly-glutamine diseases, is a dominantly inherited movement disorder characterized by formation of cytosolic and nuclear inclusion bodies and progressive neurodegeneration. Recently, we have shown that Bcl-2-associated athanogene-1 (BAG1), a multifunctional co-chaperone, modulates toxicity, aggregation, degradation and subcellular distribution in vitro and in vivo of the disease-specific mutant huntingtin protein. Aiming at future small molecule-based therapeutical approaches, we further analysed structural demands for these effects employing the $\mathrm{C}$-terminal deletion mutant $\mathrm{BAG} \Delta \mathrm{C}$. We show that disruption of the $\mathrm{BAG}$ domain known to eliminate intracellular heat shock protein 70 (Hsp70) binding and activation also precludes binding of Siah-1 thereby leaving nuclear huntingtin translocation unaffected. At the same time BAG $\Delta \mathrm{C}$ fails to induce increased proteasomal huntingtin turnover and does not inhibit intracellular huntingtin aggregation, a pre-requisite necessary for prevention of huntingtin toxicity.
\end{abstract}


Keywords: BAG1; Huntington's disease; Chaperone system; Siah1

\section{Introduction}

Bcl-2 associated athenogene 1 (BAG1) is an antiapoptotic co-chaperone of Hsp70 and its cognate protein Hsc70 (hereafter referred to as Hsp70) [1]. It binds to the ATPase domain of Hsp70 through its C-terminus. Via its N-terminal ubiquitin-like motif it was found to bind to the E3 ligase CHIP (carboxyl terminus of Hsc70-interacting protein) and to the $\alpha 7$-subunit of the proteasome [2,3]. Thus it is thought, that unfolded Hsp70 substrates are delivered by BAG1 to the proteasome for degradation. BAG1 can also bind to Raf1 kinase, thereby modulating cell growth and differentiation via the ERK pathway $[4,5]$. This binding competes with its binding to Hsp70, implying that BAG1 might act as a switch between cellular stress, growth and differentiation. In neurons, BAG1 has been shown to promote differentiation [6]; its importance for the neuronal system is underlined by the fact, that mice lacking BAG1 are lethal in utero due to massive apoptosis in the nervous system, heart and liver [7]. On the other hand, BAG1 overexpression can protect from ischemic cell death and plays an important role in axonal regeneration in vivo $[5,8]$.

Another binding partner for BAG1 is Siah1, a p53 inducible pro-apoptotic protein and E3 ligase [9] linking BAG1 to the pathogenesis of Huntington's disease. Huntington's disease (HD) is a dominantly inherited fatal neurodegenerative disease resulting from a glutamine-encoding CAG trinucleotide repeat expansion in exon 1 of the IT15 gene coding for huntingtin (htt) [10]. It is ubiquitiniously expressed, but the mutation primarily affects striatal and cortical neurons. The pathomechanisms of HD still remain unclear. It is thought that gene transcription [11-14], cellular transport [15,16], mitochondrial dysfunction, as well as impairment of the ubiquitin-proteasome system [17-19] play a critical role in the disease. One important feature of htt is the cleavage of the N-terminal glutamineexpansion containing fragment. In this regard, cleaved mutated htt enters the nucleus in complex together with GAPDH and Siah1 [20]. This translocation to the nucleus is believed to be a major step in the pathogenesis of HD [21,22]. In a previous study, we indeed were able to show, that BAG1 reduces aggregation and accelerates degradation of mutant htt in a proteasome- and Hsp70-dependent manner. Moreover, it reduces nuclear levels of mutant htt. Interestingly BAG1 downregulates cellular levels of Siah1 and overexpression of Siah-1 abolishes BAG1 effects [23].

Aiming for possible therapeutic strategies we further analysed the structural demands of BAG1. Therefore, we employed a C-terminal deletion mutant of BAG1 disrupting the functional BAG domain, through which binding to Hsp70 and Siah1 is provided.

\section{Results and Discussion}

\subsection{BAGAC is not binding and not able to downregulate Siah1}

We transiently expressed BAG1 and BAG $\Delta \mathrm{C}$ in CSM14.1 cells as shown before. By coimmunopreciptitation experiments we confirmed binding of full length BAG1 to Siah1 (Figure 1a) [23] while binding of the C-terminal deletion mutant of BAG (BAG $\Delta \mathrm{C}$ ) to Siah1 is not detectable. 
(Figure1a). As we and others could show previously, that BAG1 is inhibiting or down-regulating Siah1 in vitro, we were interested if BAG $\Delta \mathrm{C}$ could still influence Siah1 levels in cells $[9,23]$. As expected, we observed similar Siah1 levels in cells overexpressing BAG $\Delta \mathrm{C}$ when compared to controls, while Siah1 levels in presence of BAG1 were reduced (Figure 1b).

Figure 1. (a) BAG1, but not BAG $\Delta \mathrm{C}$ binds to SIAH.

a)

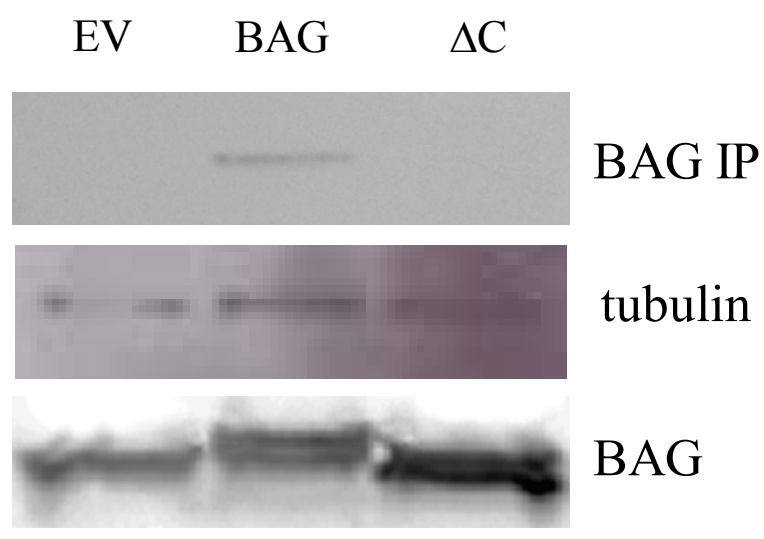

b) $\quad \mathrm{EV} \quad \mathrm{BAG} \quad \Delta \mathrm{C}$

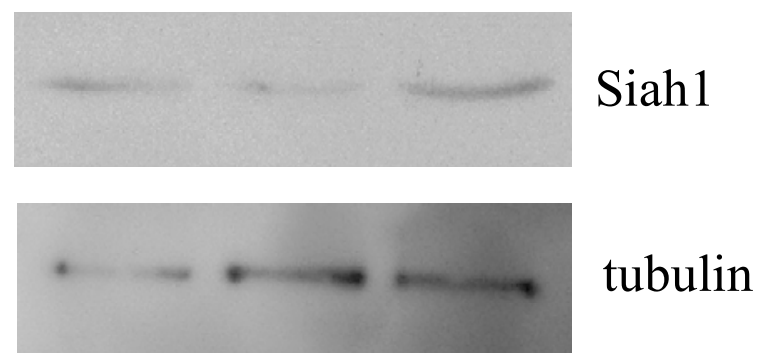

Binding was revealed by co-immunoprecipitation. CSM14.1 cells where transiently transfected with either empty vector (EV), BAG1, or BAG $\Delta \mathrm{C}(\Delta \mathrm{C}) .48 \mathrm{~h}$ after transfection cells were lysed. Pulldown was performed using an antibody directed against Siah1. Western Blots were probed with an antibody against BAG1. Note that BAG $\Delta \mathrm{C}$ is no longer able to bind Siah1 (upper panel). Staining against tubulin served as loading control (middle panel). Whole cell lysate was subjected to immunoblotting and stained with an antibody against BAG for expression analysis (lower panel). (b) BAG $\Delta$ C does not alter Siah1 levels. CSM14.1 cells were transiently transfected with either empty vector $(\mathrm{EV}), \mathrm{BAG} 1$, or BAG $\Delta \mathrm{C}(\Delta \mathrm{C}) .48 \mathrm{~h}$ after transfection cells were lysed. Whole cell lysate was subjected to western blotting and stained with an antibody against Siah1 (upper panel). Note that only BAG1 but not BAG $\Delta \mathrm{C}$ is reducing cellular Siah1 levels. Staining against tubulin served as loading control (lower panel).

\subsection{Reduction of htt inclusions}

Previous results suggested that BAG $\Delta \mathrm{C}$ acts in a dominant negative way on protein refolding [24]. Thus, we hypothesized that the C-terminal deletion mutant of BAG1 would not be able to reduce htt 
aggregate formation. To test our hypothesis, we first employed a C-terminal eGFP fused htt protein transfected to CMS14.1 cells [23]. As known from our previous study, BAG1 colocalizes to inclusion bodies and is able to reduce the amount of aggregates significantly. We then co-transfected the eGFPhtt together with BAG $\Delta \mathrm{C}$ and indeed could not find any significant reduction of inclusion bodies compared to controls (Figure 2a). To validate this data, we employed a filter retardation assay established before [25] and observed a significant reduction of htt aggregates in cells transfected with $\mathrm{BAG1}$, an effect abolished by disruption of the BAG domain in cells over-expressing BAG $\Delta \mathrm{C}$ (Figure2b).

Figure 2. BAG1, but not BAG $\Delta \mathrm{C}$ decreases mut-htt aggregation.

a)

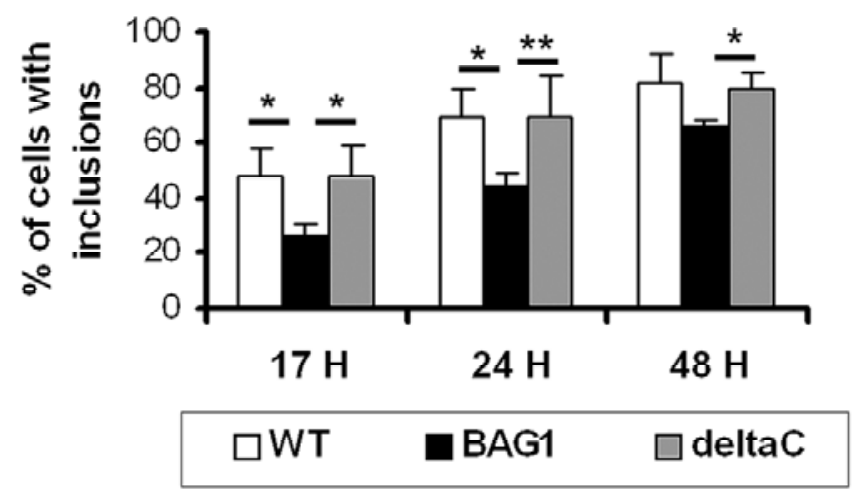

b)

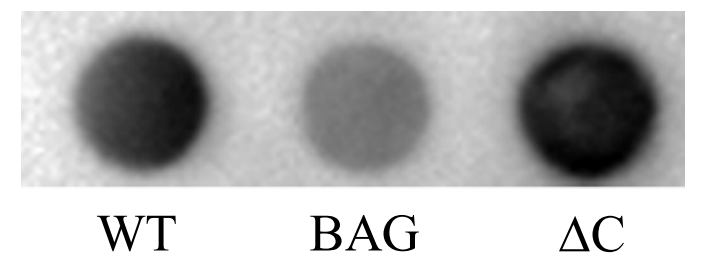

CSM $14.1 \mathrm{wt}, \mathrm{BAG} 1$ and BAG $\Delta \mathrm{C}(\Delta \mathrm{C})$ cells were transfected with htt-mut fused to eGFP. The percentage of cells that contained inclusions quantified. Cells stably expressing BAG1 are less likely to develop inclusions than wild type cells or cells stably expressing BAG $\Delta \mathrm{C}$ at all time points investigated post-transfection (a). Reduction in aggregate formation can also be observed in a filter retardation assay (b), where captured aggregates were detected with an antibody directed against the polyQ stretch. At least 400 cells were scored for each condition. The data represent mean values and SEM of three independent experiments (Student's $t$-test, ${ }^{*}, \mathrm{p}<0.05,{ }^{*}, \mathrm{p}<0.01$, a; ANOVA, ${ }^{*}, \mathrm{p}<0.05, \mathrm{~b}$ ).

\subsection{BAGAC is no longer able to enhance htt degradation}

We recently were able to show that BAG1 reduces htt aggregate formation by increasing htt turnover in a proteasome dependent manner, as BAG1 is able to interact with the $\alpha 7$-subunit of the proteasomal core [23]. To investigate whether BAG $\Delta \mathrm{C}$ is still able to influence htt-mut degradation, we performed a cycloheximide chase experiment. In contrast to BAG1, BAG $\Delta \mathrm{C}$ did not increase mut- 
htt turnover (Figure 3a). In cells stably expressing BAG1 htt-mut is cleared faster with a half-life of $\sim 3.3$ hours as compared to wild type cells in which it had a half-life of $\sim 4.9$ hours (Figure $3 b$ ). In line with previous results from our laboratory where BAG $\Delta \mathrm{C}$ appeared to have a dominant negative effect on protein folding, mutant htt seemed to be even more stable in the presence of BAG $\Delta \mathrm{C}$ (half-life $\sim 9,5$ hours) than in wild type cells. (Figure 3b) [24]. Expression levels of htt remained unaltered by the presence of BAG1 or BAG $\Delta \mathrm{C}$ (Figure 3c).

Figure 3. BAG $\Delta \mathrm{C}$ does not enhance htt-mut degradation.

a)

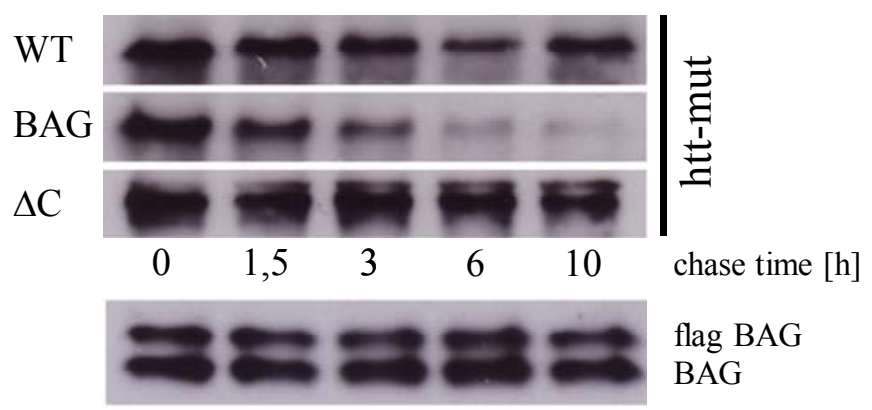

b)

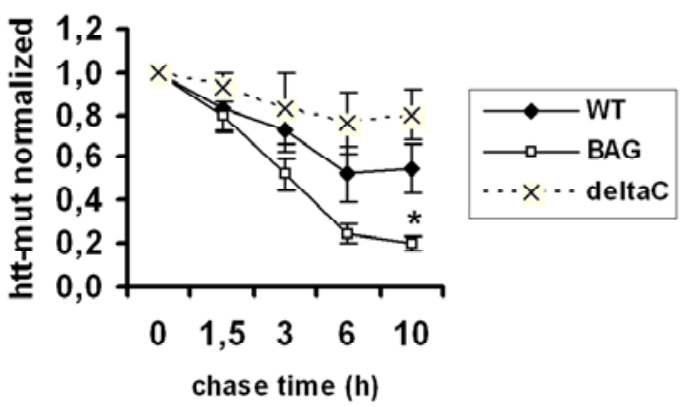

c)

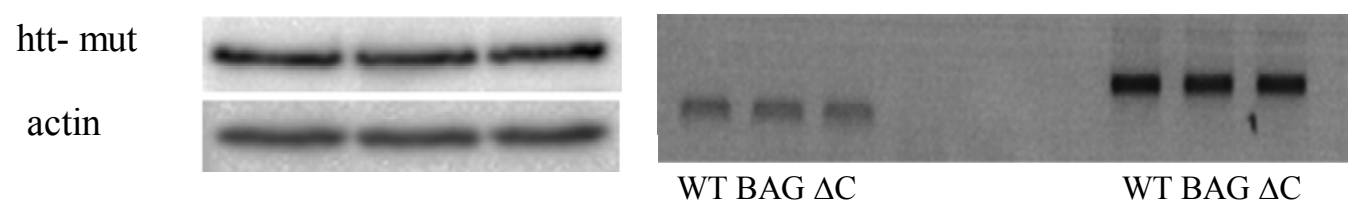

(a) CSM $14.1 \mathrm{wt}, \mathrm{BAG} 1$ or BAG $\Delta \mathrm{C}(\Delta \mathrm{C})$ cells were transiently transfected with htt-mut. $\sim 17$ hours post-transfection, cycloheximide (chx) was added at $10 \mu \mathrm{g} / \mathrm{mL}$ for indicated time periods. Htt-mut is degraded faster in BAG1 cells, as compared to wild type cells. The presence of BAG $\Delta \mathrm{C}$ stabilizes htt-mut. Endogenous BAG1 as well as the overexpressed flag-BAG1 levels do not decrease during the time course of the chx chase. (b) Three independent experiments were quantified by densitometry (b, ANOVA, *, p < 0.05; CSM 14.1 cells). (c) Expression levels of httmut protein after a transient transfection are the same among the different cell lines (left panel). mRNA levels of transiently transfected htt-mut are similar in all three cell lines as seen by RT-PCR (right panel).

\section{4. htt translocation to the nucleus is not modulated by BAG $\Delta \mathrm{C}$}

Mutant htt was found both in the cytoplasm and in the nucleus when overexpressed in CSM14.1 cells. This is also in accordance with previous studies showing nuclear accumulation leading to toxicity of the mutant protein [21,22]. In cells stably expressing BAG1, nuclear htt-mut levels were 
decreased compared to wild type cells (Figure 4 upper right panel) In contrast, over expressing $\mathrm{BAG} \Delta \mathrm{C}$ fails to reduce the amount of htt-mut inside the nucleus (Figure 4 lower right panel).

Figure 4. BAG1 influences htt-mut subcellular localization.

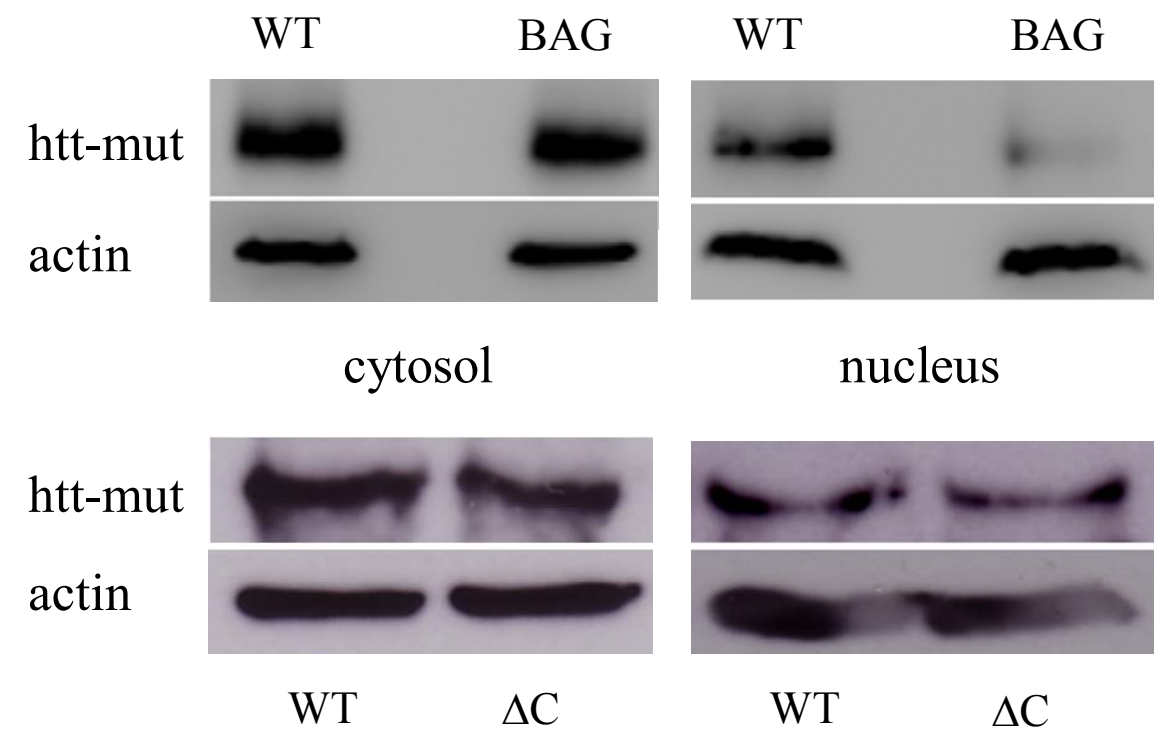

CSM 14.1 cells were transfected with htt-mut and subjected to subcellular fractionation and immunoblotting.(upper panel). Htt-mut is found both in the cytosol and nucleus. Cells stably expressing BAG1 show significantly less htt-mut within the nuclear fraction In contrast, BAG $\Delta \mathrm{C}$ $(\Delta \mathrm{C})$ does not prevent nuclear accumulation of htt-mut (lower panel).

\section{Experimental}

\subsection{Protein extract preparation}

Cells were washed, scraped in ice-cold PBS and pelleted. Cell lysates were prepared using a buffer containing $50 \mathrm{mM}$ TRIS- $\mathrm{HCl}(\mathrm{pH} 7,4), 150 \mathrm{mM} \mathrm{NaCl}, 1 \%$ Triton-X 100 and complete protease inhibitor cocktail (Roche) for 15 minutes at $4{ }^{\circ} \mathrm{C}$, followed by 10 minutes centrifugation at $13,000 \mathrm{rpm}$ at $4{ }^{\circ} \mathrm{C}$. The pellets which were further used for the filter retardation assay were resuspended in the lysis buffer and sonicated.

\subsection{Filter retardation assay}

For the filter retardation assay protein extracts were heated at $98{ }^{\circ} \mathrm{C}$ for 3 min in $2 \%$ SDS and $50 \mathrm{mM}$ DTT and filtered through a $0.2 \mu \mathrm{m}$ cellulose acetate membrane (Schleicher \& Schuell, Dassel, Germany) using a dot-blot filtration unit. The membranes were further processed for immunodetection.

\subsection{Antibodies}

The following antibodies were used at corresponding dilutions: rabbit polyclonal CAG53b, 1:2,000 (a gift from E.E, Wanker), mouse monoclonal 1C2, 1:1,000 (Chemicon), rabbit polyclonal BAG 1680

[6], 1:1,000, goat polyclonal Siah1, 1:1,000 (Everest Biotech), mouse monoclonal $\beta$-tubulin, 1:1,000 (Sigma), actin, 1:5,000, (Chemicon). 


\subsection{Immunoblotting}

For Western blotting, a Mini Trans-Blot Cell setup $(7.5 \times 10 \mathrm{~cm}$ blotting area, Bio-Rad $)$ was used. After the blotting step, the membrane was incubated in blocking solution (5\% milk in TBS-T) for 1 hour at room temperature to avoid unspecific binding of the antibody, at $4{ }^{\circ} \mathrm{C}$ overnight and with secondary HRP-conjugated antibodies for $1 \mathrm{~h}$ at room temperature. The membrane was washed $3 \times 20$ minutes with TBS-T after each antibody incubation. To develop membranes ECL was used and applied for 2 minutes onto the membrane. Films (Amersham, Hyperfilm ECL) were exposed to the membrane and developed using AGFA Curix 60 table-top processor.

\subsection{Co-immunoprecipitation}

Cell lysates were prepared as described above. Affinity beads (Sigma) were washed twice with lysis buffer and $25 \mu \mathrm{L}$ beads were added to each tube. The beads were then incubated with cellular lysates for 2 hours at $4{ }^{\circ} \mathrm{C}$ with rotation. Subsequently the pulldown anitibody directed against Siah1 was added. Lysates were again incubated at $4{ }^{\circ} \mathrm{C}$ overnight. Next they were spun down, washed twice with the lysis buffer and twice with TBS buffer ( $400 \mu \mathrm{L}, 5$ minutes each washing step). Proteins bound to the beads were eluted by adding $20 \mu \mathrm{L} 2 \mathrm{X}$ SDS sample buffer for 5 minutes at $95{ }^{\circ} \mathrm{C}$.

\subsection{Cell culture}

CSM $14.1 \mathrm{wt}$ cells were grown in DMEM supplemented with $10 \%$ FCS and penicillin/streptomycin in $32{ }^{\circ} \mathrm{C}$. Stably transfected CSM-BAG1 and CSM- $\Delta \mathrm{C}$ were maintained in the same medium with an addition of puromycin. HEK293T cells were grown in DMEM supplemented with $10 \%$ FCS and penicillin/streptomycin in $37{ }^{\circ} \mathrm{C}$. For transient transfections Lipofectamine 2000 (Invitrogen) was used.

\subsection{Transient transfections}

Most transfections were done using Lipofectamine 2000 reagent. In case when large amount of cells needed to be transfected, a more cost-efficient calcium phosphate method was used. Lipofectamine transfections were done according to the manufacturers provided protocol.

\subsection{Calcium phosphate transfection}

Cells were plated on a $10 \mathrm{~cm}$ dish, $45 \mu \mathrm{g}$ DNA was mixed with $125 \mu \mathrm{L}$ of $2 \mathrm{M} \mathrm{CaCl}_{2}$ and $\mathrm{H}_{2} \mathrm{O}$ to achieve a total volume of $1 \mathrm{~mL}$. One $\mathrm{mL}$ of $2 \times$ HEPES buffer was added, mixed and incubated for 15 minutes at room temperature. During the incubation time, fresh medium was added to the cells. Following the incubation time, the mixture was added dropwise to the cells. Twelve hours post transfection cell were washed in PBS and fresh medium was added. Gene expression was assayed 36 hours later.

\subsection{Densitometry analysis}

To quantify Western blot protein bands, ImageJ software was used. The blot images were opened in the program as tiff files. A same size rectangular selection was drawn around each band and lanes were 
selected using Analyze $>$ Gels $>$ Select Lane function. Histograms representing the intensity of pixels in the selected areas were created using Analyze $>$ Gels $>$ Plot Lanes function. The area under the histograms, representing pixel intensity in the selected area, was limited using the straight line selection in order to integrate the signal. The values of the histogram area were obtained by clicking inside the histogram with the wand tracing tool. Values corresponding to the protein of interest were normalized by dividing them by the values of the loading control to account for differences in the amount of protein loaded.

\section{Conclusions}

BAG1 is a multifunctional protein involved in central nervous system development and regeneration. It is believed to act as a switch between the proteasomal and chaperone mediated protein detoxification and is involved in cell cycle and regeneration. It was shown previously by us and others that BAG1 can ameliorate htt toxicity in vitro and in vivo $[23,26]$. These results suggested this effect to be due to increased proteasomal degradation with BAG1 acting as an accelerator of Hsp70 mediated protein refolding on the one and proteasomal degradation on the other hand [23,24]. Furthermore, we could show that BAG1 also downregulates Siah1 levels in cells thereby preventing the transfer of mutated htt into the nucleus, which is believed to be another key event in htt toxicity $[20,22,23]$. In our current study, we tried to gain more insight into the different functional parts of BAG1 and their potential therapeutical role. We therefore applied BAG $\Delta \mathrm{C}$, a C-terminal deletion mutant of BAG1 lacking the BAG domain, which is essential. for Hsp70 binding and activation [24]. We show here that this mutant is also not able to bind Siahl anymore (Figure 1a) [9]. Indeed BAG $\Delta \mathrm{C}$ also fails to downregulate Siah1 levels in vitro when compared to full length BAG1 (Figure 1b). Consequently, nuclear htt translocation is not reduced (Figure 4). This supports our previous finding that downregulation of Siah1 by BAG1 is due to a direct interaction and probably dependent on increased proteasomal degradation of Siah1, as blockage of the proteasomal system can reverse this effect [23].

Another hallmark of BAG1 is its ability to prevent mutated htt aggregation in a proteasome dependent manner in vitro [23]. This bears up the theory that BAG1 acts as a "shuttle" between the chaperone system and the proteasomal system for more rapid degradation of misfolded proteins [3]. In line with this, BAG $\Delta \mathrm{C}$ is not able to provide aggregate reduction of mutated htt (Figure 2) suggesting BAG1 as HSP70 "assistant" in binding and refolding mutated htt before shuttling it to the proteasomal system where BAG1 can accelerate protein degradation.

\section{Acknowledgements}

This study was supported by the CMPB and the High Q foundation. KS was financed by a grant from the European Commission to NEUREST, Contract No. MEST-CT-2004-504193.

\section{References and Notes}

1. Takayama, S.; Bimston, D.N.; Matsuzawa, S.; Freeman, B.C.; Aime-Sempe, C.; Xie, Z.; Morimoto, R.I.; Reed, J.C. BAG-1 modulates the chaperone activity of Hsp70/Hsc70. EMBO J. 1997, 16, 4887-4896. 
2. Luders, J.; Demand, J.; Hohfeld, J. The ubiquitin-related BAG-1 provides a link between the molecular chaperones Hsc70/Hsp70 and the proteasome. J. Biol. Chem. 2000, 275, 4613-4617.

3. Demand, J.; Alberti, S.; Patterson, C.; Hohfeld, J. Cooperation of a ubiquitin domain protein and an E3 ubiquitin ligase during chaperone/proteasome coupling. Curr. Biol. 2001, 11, 1569-1577.

4. Song, J.; Takeda, M.; Morimoto, R.I. Bag1-Hsp70 mediates a physiological stress signalling pathway that regulates Raf-1/ERK and cell growth. Nat. Cell Biol. 2001, 3, 276-282.

5. Planchamp, V.; Bermel, C.; Tonges, L.; Ostendorf, T.; Kugler, S.; Reed, J.C.; Kermer, P.; Bahr, M.; Lingor, P. BAG1 promotes axonal outgrowth and regeneration in vivo via Raf-1 and reduction of ROCK activity. Brain 2008, 131, 2606-2619.

6. Kermer, P.; Krajewska, M.; Zapata, J.M.; Takayama, S.; Mai, J.; Krajewski, S.; Reed, J.C. Bag1 is a regulator and marker of neuronal differentiation. Cell Death Differ. 2002, 9, 405-413.

7. Gotz, R.; Wiese, S.; Takayama, S.; Camarero, G.C.; Rossoll, W.; Schweizer, U.; Troppmair, J.; Jablonka, S.; Holtmann, B.; Reed, J.C.; Rapp, U.R.; Sendtner, M. Bag1 is essential for differentiation and survival of hematopoietic and neuronal cells. Nat. Neurosci. 2005, 8, 1169-1178.

8. Kermer, P.; Digicaylioglu, M.H.; Kaul, M.; Zapata, J.M.; Krajewska, M.; Stenner-Liewen, F.; Takayama, S.; Krajewski, S.; Lipton, S.A.; Reed, J.C. BAG1 over-expression in brain protects against stroke. Brain Pathol. 2003, 13, 495-506.

9. Matsuzawa, S.; Takayama, S.; Froesch, B.A.; Zapata, J.M.; Reed, J.C. p53-inducible human homologue of Drosophila seven in absentia (Siah) inhibits cell growth: suppression by BAG-1. EMBO J. 1998, 17, 2736-2747.

10. Group, H.s.D.C.R. A novel gene containing a trinucleotide repeat that is expanded and unstable on Huntington's disease chromosomes. The Huntington's Disease Collaborative Research Group. Cell 1993, 72, 971-983.

11. Dunah, A.W.; Jeong, H.; Griffin, A.; Kim, Y.M.; Standaert, D.G.; Hersch, S.M.; Mouradian, M.M.; Young, A.B.; Tanese, N.; Krainc, D. Sp1 and TAFII130 transcriptional activity disrupted in early Huntington's disease. Science 2002, 296, 2238-2243.

12. Wyttenbach, A.; Swartz, J.; Kita, H.; Thykjaer, T.; Carmichael, J.; Bradley, J.; Brown, R.; Maxwell, M.; Schapira, A.; Orntoft, T.F.; Kato, K.; Rubinsztein, D.C. Polyglutamine expansions cause decreased CRE-mediated transcription and early gene expression changes prior to cell death in an inducible cell model of Huntington's disease. Hum. Mol. Genet. 2001, 10, 1829-1845.

13. Zhai, W.; Jeong, H.; Cui, L.; Krainc, D.; Tjian, R. In vitro analysis of huntingtin-mediated transcriptional repression reveals multiple transcription factor targets. Cell 2005, 123, 1241-1253.

14. Zuccato, C.; Tartari, M.; Crotti, A.; Goffredo, D.; Valenza, M.; Conti, L.; Cataudella, T.; Leavitt, B.R.; Hayden, M.R.; Timmusk, T.; Rigamonti, D.; Cattaneo, E. Huntingtin interacts with REST/NRSF to modulate the transcription of NRSE-controlled neuronal genes. Nat. Genet. 2003, 35, 76-83.

15. Gunawardena, S.; Her, L.S.; Brusch, R.G.; Laymon, R.A.; Niesman, I.R.; Gordesky-Gold, B.; Sintasath, L.; Bonini, N.M.; Goldstein, L.S. Disruption of axonal transport by loss of huntingtin or expression of pathogenic polyQ proteins in Drosophila. Neuron 2003, 40, 25-40. 
16. Szebenyi, G.; Morfini, G.A.; Babcock, A.; Gould, M.; Selkoe, K.; Stenoien, D.L.; Young, M.; Faber, P.W.; MacDonald, M.E.; McPhaul, M.; Brady, S.T. Neuropathogenic forms of huntingtin and androgen receptor inhibit fast axonal transport. Neuron 2003, 40, 41-52.

17. Bennett, E.J.; Shaler, T.A.; Woodman, B.; Ryu, K.Y.; Zaitseva, T.S.; Becker, C.H.; Bates, G.P.; Schulman, H.; Kopito, R.R. Global changes to the ubiquitin system in Huntington's disease. Nature 2007, 448, 704-708.

18. Verhoef, L.G.; Lindsten, K.; Masucci, M.G.; Dantuma, N.P. Aggregate formation inhibits proteasomal degradation of polyglutamine proteins. Hum. Mol. Genet .2002, 11, 2689-2700.

19. Jana, N.R.; Zemskov, E.A.; Wang, G.; Nukina, N. Altered proteasomal function due to the expression of polyglutamine-expanded truncated N-terminal huntingtin induces apoptosis by caspase activation through mitochondrial cytochrome c release. Hum. Mol. Genet. 2001, 10, 1049-1059.

20. Bae, B.I.; Hara, M.R.; Cascio, M.B.; Wellington, C.L.; Hayden, M.R.; Ross, C.A.; Ha, H.C.; Li, X.J.; Snyder, S.H.; Sawa, A. Mutant huntingtin: nuclear translocation and cytotoxicity mediated by GAPDH. Proc. Natl. Acad. Sci. USA 2006, 103, 3405-3409.

21. Saudou, F.; Finkbeiner, S.; Devys, D.; Greenberg, M.E. Huntingtin acts in the nucleus to induce apoptosis but death does not correlate with the formation of intranuclear inclusions. Cell 1998, 95, 55-66.

22. Peters, M.F.; Nucifora, F.C., Jr.; Kushi, J.; Seaman, H.C.; Cooper, J.K.; Herring, W.J.; Dawson, V.L.; Dawson, T.M.; Ross, C.A. Nuclear targeting of mutant Huntingtin increases toxicity. Mol. Cell Neurosci. 1999, 14, 121-128.

23. Sroka, K.; Voigt, A.; Deeg, S.; Reed, J.C.; Schulz, J.B.; Bahr, M.; Kermer, P. BAG1 modulates huntingtin toxicity, aggregation, degradation, and subcellular distribution. J. Neurochem. 2009, 111, 801-807.

24. Liman, J.; Ganesan, S.; Dohm, C.P.; Krajewski, S.; Reed, J.C.; Bahr, M.; Wouters, F.S.; Kermer, P. Interaction of BAG1 and Hsp70 mediates neuroprotectivity and increases chaperone activity. Mol. Cell Biol. 2005, 25, 3715-3725.

25. Wanker, E.E.; Scherzinger, E.; Heiser, V.; Sittler, A.; Eickhoff, H.; Lehrach, H. Membrane filter assay for detection of amyloid-like polyglutamine-containing protein aggregates. Methods Enzymol. 1999, 309, 375-386.

26. Jana, N.R.; Dikshit, P.; Goswami, A.; Kotliarova, S.; Murata, S.; Tanaka, K.; Nukina, N. Cochaperone CHIP associates with expanded polyglutamine protein and promotes their degradation by proteasomes. J. Biol. Chem. 2005, 280, 11635-11640.

Sample Availability: Comercially available.

(C) 2010 by the authors; licensee MDPI, Basel, Switzerland. This article is an open access article distributed under the terms and conditions of the Creative Commons Attribution license (http://creativecommons.org/licenses/by/3.0/). 